\title{
Image Fusion Based on Wavelet Transformation
}

\author{
Raghawendra Bhimarao Naik, Pavan N.Kunchur
}

\begin{abstract}
The article based totally on the MATLAB software program simulation was carried out on the image fusion; to design and develop a MATLAB based image processing application for fusing two images of the similar scene received through other modalities. The application is required to use Discrete Wavelet Transform (DWT) and Pulse Coupled Neural Network (PCNN) techniques. The comparison is to be performed on the results obtained on the above mentioned techniques.
\end{abstract}

Keywords: MATLAB; wavelet transform; the Pulse Coupled Neural Network method; image fusion.

\section{INTRODUCTION}

The outstanding combination of pictures captured since exclusive modalities or instruments is of huge significance in numerous functions like medicinal imaging, microscopic imaging, far-off sensing PC vision and robotics .Image fusion are often described because the method by using which quite a few pictures or a number of their points are combined collectively to make one image. Image fusions are often achieved by extraordinary stages of the knowledge demonstration 4 unique degrees are often distinct consistent with Abidi and Gonzals signal pixel characteristic and representational stages. So far the effect of image fusion in fields like far-off realize and clinical images are generally meant used for arrangement in the direction of a person's viewer intended for simpler with most suitable explanation. Accordingly the understanding of the combined picture is of dominant significance while deciding one-of-a-kind fusion schemes. A few universal necessities are regularly demanded on the fusion resulting the combined picture need to maintain as carefully as viable all applicable data contained inside the input pics the fusion method will not set up any works or inconsistency which might divert otherwise misinform the individual spectator or some successive image giving out steps. Once fusion is completed on pixel stage the participation pictures are united with none preprocessing. Pixel stage fusion algorithms differ as of extremely simpler picture standardizing in the direction of especially complicated example major element evaluation PCA pyramid based image fusion and wavelet transform fusion. Some techniques near pixel level fusion are regularly special counting on whether or not the pictures are combined within the spatial area otherwise during a change area. Once the combined picture is achieved it's going to exist transformed similarly with a few elements of activity could also be obtained.

Revised Manuscript Received on June 05, 2020.

* Correspondence Author Engineering, KLS Gogte Institute of Technology, Belagavi. E-mail: Raghawendranaik@gmail.com

Pavan N.Kunchur, Assistant Professor, Department of Computer Science and Engineering, Gogte Institute of Technology, Belagavi. E-mail: pnkunchur@git.edu

(C) The Authors. Published by Blue Eyes Intelligence Engineering and Sciences Publication (BEIESP). This is an open access article under the CC BY-NC-ND license (http://creativecommons.org/licenses/by-nc-nd/4.0/)
Raghawendra Bhimarao Naik*, Department of Computer Science and

\section{LITERATURE SURVEY}

Discrete wavelet transforms (DWT) [3], [4] based image fusion be first amongst the mainly important easiest pretty picture synthesis. The primary stair within picture fusion be that the multi scale disintegration of origin pictures. The supply pictures are separated interested in low and better associate bands. The pixel holding biggest wavelet coefficients are chosen for function. DWT's behaves an exchange of photograph in spatial area headed for picture into occurrence field. The fusion operator wormed within this technique differs in support of distinctive disintegration stages. The main benefit through the use of DWT's is to facilitate it conserves coefficient statistics for the reason that it use distinctive combination guidelines thus it grant higher (SNR). The remaining tread inside DWT's based totally approach is that the utility of inverse discrete wavelet transform in the direction of the practiced picture.

Wang, Wencheng along with Chang, Faliang [5], propose a multi-focus picture combination approach supported Laplacian pyramid. Image fusion primarily based under laplacian pyramid is the usage of pyramid based disintegration. The participation picture may destroy down interested in distinctive pyramid stages by laplacian pyramid. The benefit of these decomposition schemes is it properly conserves part records into picture. Mainly this laplacian pyramid is resulting starting a small omit gaussian pyramid with recursive filtering method. This technique include 2 pyramid stage conversations: fundamental structure of gaussian pyramid formation, arrangement laplacian pyramid since gaussian pyramids. Similar to DWT's primarily based combination this get entry to additionally makes use of wonderful drivers to combine one-of-a-kind pyramid stages and in the end makes use of an inverse laplacian pyramid convert to rebuild the combined outcome. The algorithm taken on behalf of this technique is not a good deal advantageous as traditional techniques however the most benefit be it knows how to observe shade in picture.

$\mathrm{Li}$, Shutao along with Kwok, JT-Y with Tsang, Ivor W as well as Wang, Yaonan [6], planned fusing pictures by distinctive focus the use of assist vector equipment. Image fusion the use of Support Vector Machine is any other technique used for picture combination which be predicated under each wavelets and machine learning advance. It desires each SVM and discrete wavelet frame transforms (DWFT) for combination reason. (DWFT) decomposes and take out function coefficients. An improvement within DWT primarily based technique is DWFT. The foremost change of DWFT as of DWT is with the purpose of it gives a conversion invariant picture giving out and illustration sample.

Published By:

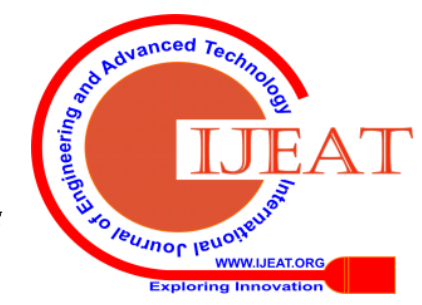




\section{Image Fusion Based on Wavelet Transformation}

A SVM is skilled used for judgment the easiest picture pixels to facilitate go well with meant for handing out. By means of DWFT as well as SVM form a few difficult wavelet coefficients are developed. This SVM primarily based technique contains the benefits of each wavelet disintegration with SVM. Dissimilar strategies like choose greatest, subjective common technique are presented meant for combine the DWFT coefficients. Based under action stages on every pixel within rotting stages, SVM acts supervised or unsupervised learning. The most important benefits of this approach be that the utilize of greater DWFT as an alternative than easy wavelets with a useful SVM primarily based strategy be employed as a replacement for than easy coefficient combining scheme. Similar to easy wavelet based totally strategies the rebuilding method be accomplished in making use of an inverse wavelet transform under pictures.

A SVM be skilled intended for discovery the easiest picture pixel to go well with for giving out. By DWFT with SVM form a few difficult wavelet coefficients be caused. This SVM primarily depended technique contains the benefits of each wavelet disintegration with SVM. Dissimilar strategies like choose greatest, biased common technique are accessible used for linking the DWFT coefficients. Depended on top of action stages on every pixels inside rotting stages, SVM behaves supervised or unsupervised learning. The most important benefits of this approach be that the utilize of greater DWFT in its place than easy wavelets with an efficient SVM primarily depended strategy. be employed as an alternative than easy coefficient combining scheme. Similar to easy wavelet located totally strategies the rebuilding method be accomplished through making use of an inverse wavelet transform under pictures.

Liang, Junli as well as He, Yang with Liu, Ding along with Zeng, Xianju, [7], recommend an Image fusion the use of greater order singular price decomposition. HOSVD is one extra statistics pushed picture fusion system. The type differentiation of HOSVD beginning additional combination systems be it dissolves the key picture interested in exclusive tensors alternatively than decrease with superior sub band in wavelet decomposition. Picture is in the beginning separated interested in distinct portions intended for characteristic withdrawal. This form of disintegration be relatively efficient into large dimensional records with into medium primarily developed operations. Originally picture be split interested in portions along with complete standards of pixels strength standards be full. Coefficient merging technique utilized into this technique is a particular sigmoid task. Reduction things control this sigmoid task. This sigmoid purpose begins when selected most system or averaging or else smoothing function supported reduction restriction. By potential of some individual of these sigmoid features remaining combined photo be achieved. These changeable sigmoid characteristics make this technique useful designed for combination of several pictures.

Local edge-preserving multi scale decomposition used for top energetic vary picture quality map [10], propose a absolutely special area retaining filter referred to as Local Edge Preserving Filter (LEP). Similar to advised picture filter LEP filter as well applies nearby linear form in the direction of get combined outcome. LEP filter delivers improved outcome than advised picture filter considering the fact that it makes use of adaptive linear coefficients inside the neighborhood small form. The linear coefficients vary through reference in the direction of every transom in use for process. Grade of pictures be in use designed intended for determine out the linear coefficients. This make LEP pass through a filter extra bendy than guided filter. The fusion method in [8], be capable of make higher consequences through changing the guided filter with LEP filter.

\section{IMAGE FUSION METHODOLOGY}

The most important levels of the universal pixel-level image fusion techniques be specified in determine 1 as well as they are 1 . Arithmetical radically change be utilized toward the key pictures on the way to achieve the change coefficients 2 . Fusion method be utilized toward construct the combined coefficients in addition to 3 . Inverse transform on the combined coefficients toward make the last combined picture.

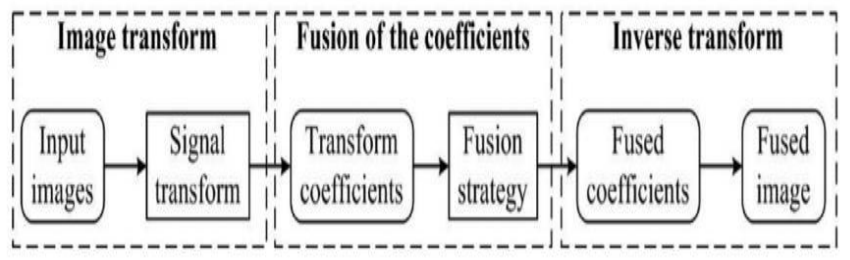

\section{Figure- 1: Outline of the primary phases used for a fundamental pixel level Image Fusion plan.}

\section{DISCRETE WAVELET TRANSFORM (DWT)}

Wavelets be situations developed as of individual particular feature through dilations and explanations. The unique design of the wavelet convert is toward make out some random feature since a superposition of wavelet. All such superposition decompose the particular feature interested in one of a kind extent stages wherever every stage be similarly decayed by a decision modified thereto stage. The DWT's be presently comparable toward a hierarchic associate band's plan anywhere the associate band's be logarithmically area in incidence with signify octave-band's disintegration. in making use of DWT's, the picture be really separated, that is decomposed as 4 associate band's within the figure below picture disintegration. A 1 stage, $\mathrm{B}$ two stage band as well as importantly associate sampling. These 4 sub band show up for the reason that separable functions of perpendicular with parallel filters. The associate band's label $\mathrm{LH}_{1}, \mathrm{HL}_{1}$ and $\mathrm{HH}_{1}$ symbolize the perfect level wavelet coefficients, that is, examine pictures even as the associate band's $\mathrm{LL}_{1}$ coincides toward frequent stage coefficient, that estimate picture.

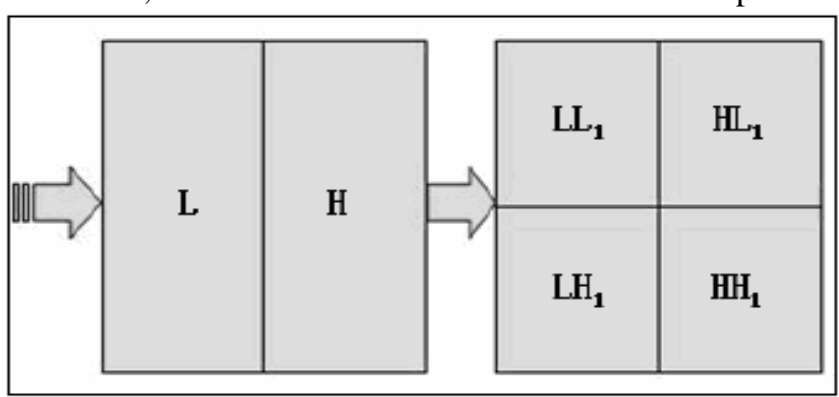

Figure-2: Image Decomposition Schematic figure
Published By:

Blue Eyes Intelligence Engineering \& Sciences Publication

(C) Copyright: All rights reserved. 


\section{Using 2-Dimensional Discrete Wavelet Transform} To accomplish subsequent frequent degree of wavelet coefficients, the associate band's $\mathrm{LL}_{1}$ solely be enhance decayed and vitally patterned. This fallout inside 2 stage wavelet breakdown. Likewise, to get extra decomposition, LL2 is use. These method remains' pending some last level is arrived. The standards or changed coefficients in difficult calculation and element pictures (associate-band's pictures) be necessary skin, which be helpful used for image fusion.

\subsection{IMAGE FUSION}

while the wavelet coefficients have massive absolute values consist of the knowledge about the huge aspects of the pictures like limits and features, an honest combination regulation be toward require get the most (complete costs) of the equivalent wavelet coefficient. The mass define of a picture combination shape be exposed inside Figure. 3 Now, wavelet convert be preliminary beneficial under input pictures. After that the wavelet coefficients of associate $B$. pictures (the distinct associate band's by unlike systems) be combined the use of combination policy. Lastly, the fused picture be got through means of making use of the inverse DWT under the combined wavelet coefficients. Here it has 2 sorts of combination policies used for combing the associate-pictures, while, 1) Pixel-based along with 2) Region-based. During pixel-based combination, pixel-bypixel wavelet coefficients be combined, each through choosing the greatest (or smallest amount) price of the equal co-efficient inside the 2 associate-pictures, or else by means of deciding on the weighted coefficient of the 2 associatepictures.

Here region-based system, the combination law be grounded totally under the numerical volume of constrained window of dimension $\mathrm{N} \times \mathrm{N}$. now, the numerical portions of each and every picture area more three $\times 3$ window be figure out while an undertaking size related by the pixel founded inside the transform.

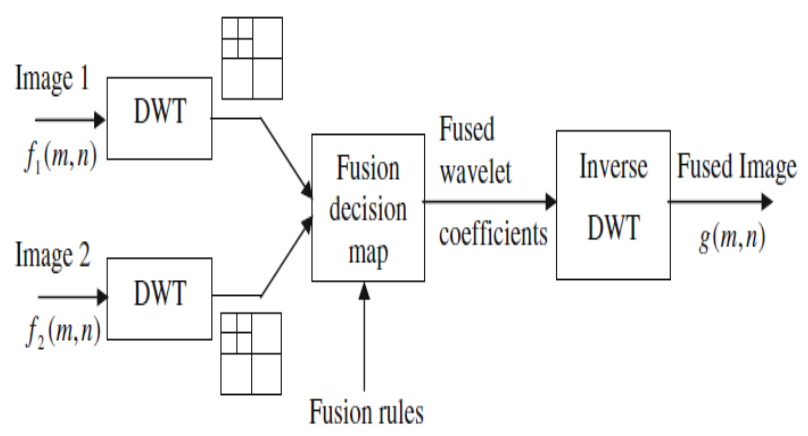

Figure 3: Block diagram of DWT

The fusion ruling be that but the motion dimension of 1 gap inside picture-1 be large (otherwise lesser) than the correspondent gap in picture-2, next the cost of the middle pixel of the gap inside picture- 1 are travelling live measured because the original pixel cost. The regularly accepted exercise dimensions are common cost, variance with power. The image fusion, projected at this factor be distinctive like pursues. The input pictures are important exposed toward 2 stages of DWT's disintegration. The wavelet coefficient inside small incidence associate-band's, that has both $\mathrm{LL}_{1}$ (of the principal picture) or $\mathrm{LL}_{2}$ (of the 2nd picture) be special supported the mixed part facts inside the corresponding excessive occurrence associate band's, that has, $\mathrm{LH}_{1}, \mathrm{HL}_{1}$ with $\mathrm{HH}_{1}$ otherwise $\mathrm{LH}_{2} \mathrm{HL}_{2}$ as well as $\mathrm{HH}_{2}$. Now, mean along with variance over three $\times 3$ window be needed because action dimension toward are looking for out the sting records current inside the excessive frequency subbands. The three $\times$ three windows inside the large incidence associate-band's be situated on the equivalent pixel of $\mathrm{LL}_{2}$. here cases, the place the undertaking procedures don't fulfill the principle, known into step-2 of the algorithm, the usual common of wavelet coefficients inside $L_{12}$ with $L_{2} L_{22}$ be capable of also survive in use. here calculation, the wavelet coefficients inside larger incidence associate-band, that has, both $\mathrm{LH}_{1} \mathrm{M}$ otherwise $\mathrm{LH}_{2} \mathrm{M}, \mathrm{HL}_{1} \mathrm{M}$ or else $\mathrm{HL}_{2} \mathrm{M}$ as well as $\mathrm{HH}_{1} \mathrm{M}$ or else $\mathrm{HH}_{2} \mathrm{M}(\mathrm{M}=1,2$ produces the quantity of DWT's disintegration) are going to survive will be selected or else mixed supported variance primarily developed under standard deviation since exercise quantify. Then, the remaining last fused picture is received through making use of the inverse DWT on the combined wavelet coefficients.

\section{PULSE COUPLED NEURAL NETWORK}

Component, $\mathrm{N}=$ number of nodes of input $(\mathrm{n} \sim 5$ here), hj=threshold of the jth unseen node. Neural networks (NN) are usually working in a variety of applications. It's practical used for arrangement with outcomes within comparable otherwise greater efficiencies as of less coaching sampling. It's a few benefits more than regular classifiers thanks toward due to 2 necessary uniqueness: their non-parametric environment as well as consequently the non-Gaussian distribution guess.

Pulse coupled neural network (PCNN) be titled the third technology $\mathrm{N}$ due to the fact it's the successive choices: (i)universal excellent approximation nature with constructive arrangement capacity; ii) fast meeting of studying system; iii) an great community toward gain the map feature internal the feed-forward; iv) rejection have just before be compelled to pre-train. Thus, throughout this in this piece of writing, we there a totally special fusion method supported PCNN. Here live on a 1-to-1 connection amongst the picture pixel as well as system neurons, which suggests, every pixels be said to a singular neurons with consequently the other way around.

Basic PCNN neurons consist of 3 elements: the accessible area, the accent area with therefore the heartbeat producer. This is frequently exposed in Figure-4.

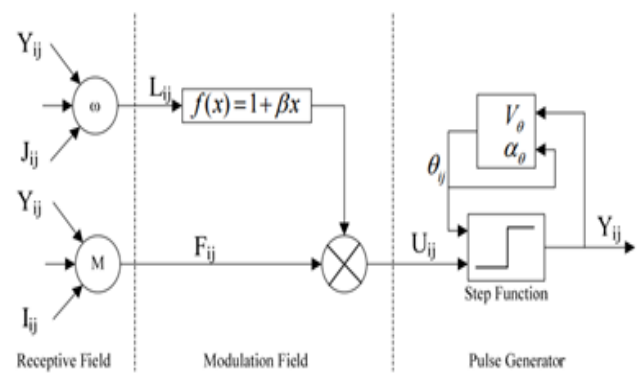

Figure-4: Components of PCNN neuron

Assume NP be that the sum variety of iteration also $\mathrm{N}$ be modern iterations, the neuromime of PCNN are often defined through way of the subsequent equation.

Published By: \& Sciences Publication

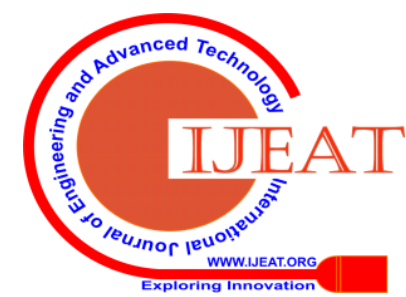




\section{Image Fusion Based on Wavelet Transformation}

$$
\begin{gathered}
F_{i j}[n]=\exp \left(-\alpha_{F}\right) F_{i j}[n-1]+V_{F} \sum_{m_{i j k} Y_{k j}[n-1]+I_{i j}} \\
L_{i j}[n]=\exp \left(-\alpha_{L}\right) L_{i j}[n-1]+V_{L} \sum_{Q_{i j k}} Y_{k j}[n-1] \\
U_{i j}[n]=F_{i j}[n]\left[1+\beta L_{i j}[n]\right) \\
Y_{i j}[n]= \begin{cases}1, & U_{i j}[n]>\theta_{i j}[n-1] \\
0, & U_{i j}[n] \leq \theta_{i j}[n-1]\end{cases} \\
\theta_{i j}[n]=\exp \left(-\alpha_{\theta}\right) \theta_{i j}[n-1]+V_{\theta i j} Y_{i j}[n-1]
\end{gathered}
$$

Wherever the $\{i, j\}$ couples provides the point of a neurons. F, L, U, Y and $\theta$ be finding keys, involving input's, inside action, pounding outcome, with active entry, correspondingly. $\alpha \mathrm{F}, \alpha \mathrm{L}$ with $\alpha \theta$ be instance stables intended for finding, connecting with active entry. VF, VL as well as $\mathrm{V} \theta$ are assigning stable, $\mathrm{M}$ and $\omega$ be the synaptic loads, and Iij with Jij be exterior input's. $\beta$ be the rule of the relating. First of all, the neurons $\{\mathrm{i}, \mathrm{j}\}$ gain inputs alerts beginning different neuron's with starting outdoor authority via the accessible areas. After that the sign is detached interested in 2 channel's. Single be feed channels (F), the opposite be connecting channels (L). Secondlly, within the accent section the connecting inputs $L$ be biased by $\beta$ with brought a continuing unfairness, after that increased by the feed inputs $F$. The inner achievement $U$ be with the reason of the outcome of the intonation element. Lastly, inside the pulse producer section $\mathrm{U}$ measures by the edges $\theta$. but $\mathrm{U}$ are better than $\theta$, the neurons determination launch a beat. If not it resolve no longer produce. Y's is the outcome. If the neuron has burned, $\theta$ will increase; in any other case $\theta$ will decay.

\section{THE ALGORITHM OF IMAGE FUSION}

\section{A. Discrete wavelet transforms:}

Step-1: cover DWT breakdown to inputs pictures, $f 1(m, n)$ with $f 2(m, n)$.

Step-2: used for every pixel of $\operatorname{LL}_{2}{ }_{2}(M, N)$ (that is estimate or short occurrence associate band's), perceive three $\times 3$ window's $\mathrm{CLH}^{k}$ two $(M, N), \mathrm{CHL}^{k} 2(m, n)$ with $\mathrm{CHH}^{k}{ }^{2}(M$, $N)$, founded on the equivalent pixels inside $\mathrm{LH}^{k}(M, N), \mathrm{HL}^{k}$ ${ }^{2}(M, N)$ and $\operatorname{HH}^{k}{ }^{2}(M, N)$ associate-band's (that has aspect otherwise high-frequency associate band), the place $K=1$, 2 additionally characterize the pictures concerned within combination.

1. Find $\mathrm{CA}^{k} 2(M, N)=\mathrm{ab}^{\prime}\left\{\mathrm{CLH}^{k}{ }^{2}(M, N)\right\}+\mathrm{ab}$ 's $\left\{\mathrm{CHL}^{k}\right.$ two $(M, N)\}+$ ab's $\left\{\mathrm{CHH}^{k} 2(M, N)\right\}$

2. Find the mean $\mathrm{MA}^{k 2}(M, N)$ with preferred deviation $S A^{k 2}(M, N)$ of $C^{k 2}(M, N)$ as undertaking stages.

3. Fuse wavelet coefficients of $\mathrm{LL}_{2}$ via the combination regulation like follow's:

$F(m, n)$

$$
= \begin{cases}\mathrm{LL}_{2}^{1}(m, n) ; & \begin{array}{r}
\text { if } \mathrm{MA}_{2}^{1}(m, n)>\mathrm{MA}_{2}^{2}(m, n) \\
\text { andSA }
\end{array} \\
\mathrm{LL}_{2}^{2}(m, n) ; & \text { if } \mathrm{MA}_{2}^{1}(m, n)>\mathrm{SA}_{2}^{2}(m, n) \\
& \text { and } \mathrm{SA}_{2}^{1}(m, n)<\mathrm{SA}_{2}^{2}(m, n) \\
\left\{\mathrm{LL}_{2}^{1}(m, n)\right. & \\
\left.+\mathrm{LL}_{2}^{2}(m, n)\right\} / 2 ; & \text { Otherwise }\end{cases}
$$

Step-3: used for every pixel of $\operatorname{LHkm}(M, N)$ (that has, excessive incidence associate band's),discover the excellent difference, SDkm over three $\times 3$ window's, were $K=1,2$ signify the pictures concerned inside fusion and $M=1$, two produces the degree of DWT decomposition. Fuse the wavelet coefficients of LHKM $(M, N)$ associate-band's the usage of the combination law since follows':

$$
\begin{aligned}
& F(m, n)
\end{aligned}
$$

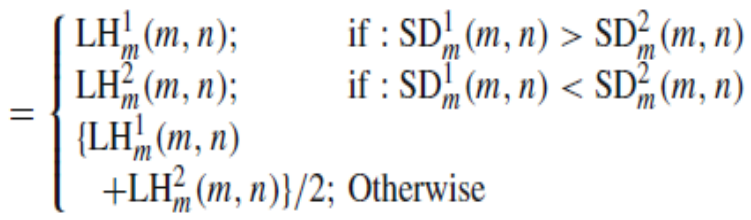

Step-4: do again step three used for additional excessive incidence associate band's

Step-5: Apply Inverse DWT toward obtain the fused picture.

\section{B. Pulse Coupled Neural Network:}

According in the direction of the next step's operating the fusion choice:

Step-1: Initialization: assume, ,Ik A ij with i k B, ij , $\{\mathrm{I}, \mathrm{j}\}$ is produced the grey of pixels inside the wide variety $\mathrm{K}$ couple sub picture, plus create a normalization toward the vary of $0-1$; assume, the preliminary price of inside link import medium, inner motion medium with threshold matrix correspondingly be:

$$
L_{v}^{k}(0)=U_{c}^{k}(0)=0, \theta_{y}^{k}(0)=1,
$$

Next to this instance, every of the neuron's be inside the fire not in situation $\mathrm{T}_{\mathrm{ij}}^{\mathrm{k}}(0)=0, \mathrm{~N}_{\max }$ be the maximal iterative period, explosion period file medium $\mathrm{T}_{\mathrm{ij}}^{\mathrm{k}}(0)=0$;

Step-2: According in the direction of the $\mathrm{L}_{\mathrm{ij}}^{\mathrm{k}}(\mathrm{n}), \mathrm{U}_{\mathrm{ij}}^{\mathrm{k}}(\mathrm{n})$, $\Theta^{\mathrm{k}}{ }_{\mathrm{ij}}(\mathrm{n})$ with $\mathrm{Y}_{\mathrm{ij}}^{\mathrm{k}}(\mathrm{n})$ The import of each and each instance iterative process used for the accumulative network be: $\mathrm{T}^{\mathrm{k}}{ }_{\mathrm{ij}}(\mathrm{n})=\mathrm{T}_{\mathrm{ij}}^{\mathrm{k}}(\mathrm{N}-1)+\mathrm{Y}^{\mathrm{k}}{ }_{\mathrm{ij}}(\mathrm{n})$

Step 3: do again the step 2. And step 3. Till $\mathrm{n}==\mathrm{N}_{\max }$, on this instance, the network iterative process is closed;

Step 4: choose wavelet coefficient of fusion picture.

$$
D_{k, F}^{k}(i, j)=
$$

$$
\begin{aligned}
& \left|\frac{D_{k, \mathrm{~A}}^{e}(i, j)+D_{k, \mathrm{~B}}^{k}(i, j)}{2},\right| T_{i, \mathrm{~A}}^{k}\left(N_{\max }\right)-T_{i, \mathrm{~B}}^{k}\left(N_{\max }\right) \mid \leq \varepsilon . \\
& D_{i, \mathrm{~A}}^{\epsilon}(i, j), \begin{array}{l}
\left|T_{\dot{j} \mathrm{~A}}^{k}\left(N_{\max }\right)-T_{i j \mathrm{~B}}^{k}\left(N_{\max }\right)\right|>\varepsilon, \\
\text { and } T_{i, \mathrm{~A}}^{k}\left(N_{\max }\right) \geq T_{i j, \mathrm{~B}}^{k}\left(N_{\max }\right)
\end{array} \\
& D_{k \mathrm{~B}}^{k}(i, j), \begin{array}{l}
\left|T_{i, \mathrm{~A}}^{k}\left(N_{\max }\right)-T_{i, \mathrm{~B}}^{k}\left(N_{\max }\right)\right|>\varepsilon, \\
\text { and } T_{\dot{\xi}, \mathrm{A}}^{k}\left(N_{\max }\right)<T_{i, \mathrm{~B}}^{k}\left(N_{\max }\right)
\end{array}
\end{aligned}
$$

Step 5: adopt uniformity inspection toward standardize the outcomes received via step-5, but neural network distinguish a positive area on or after picture a, with its nonessential area beginning picture $b$, hence the positive area pixel would survive modified through the pixel inside the equivalent area of picture b;

Step 6: lastly, rebuild the wavelet on the way to get the last fusion picture.

\section{THE FUSION RESULT}

Use of matlab wavelet transform used for the algorithm, Laplace pyramid algorithm, the outcome is as follow: 


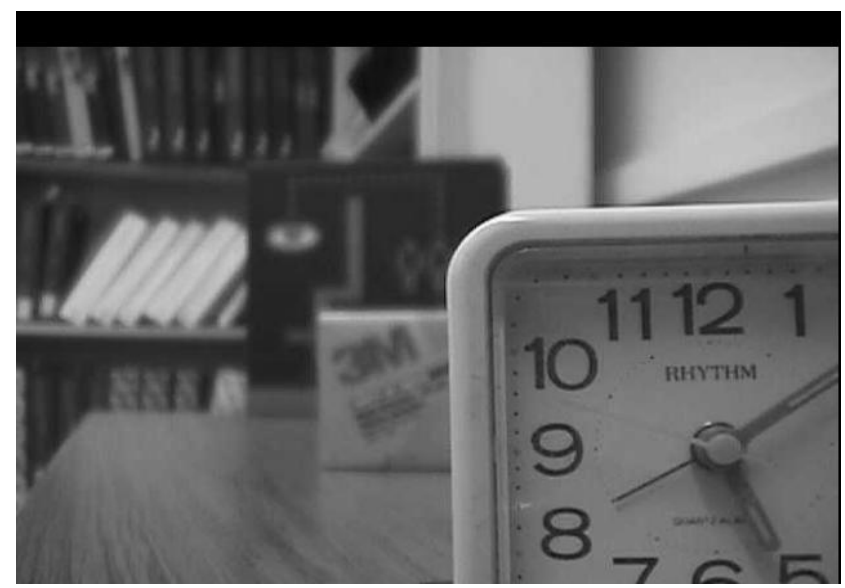

Figure-5: Input image a

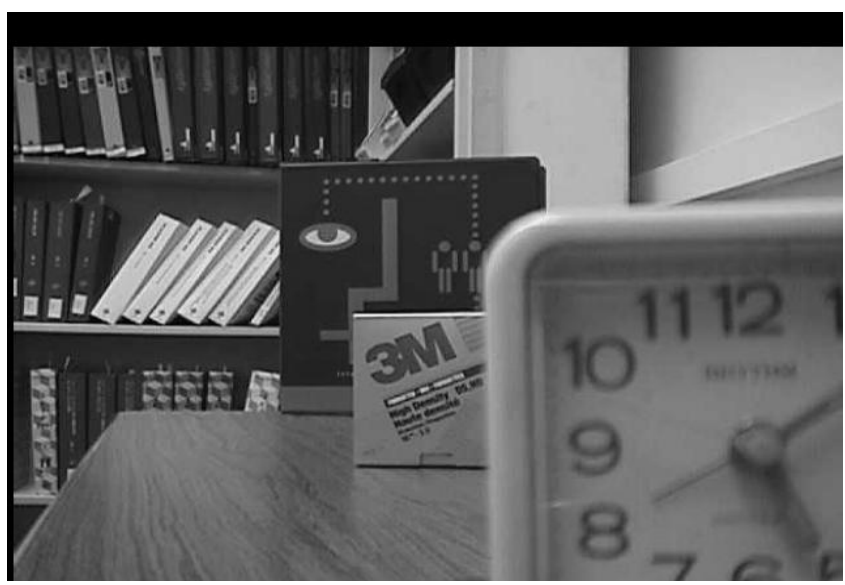

Figure 6: Input image b

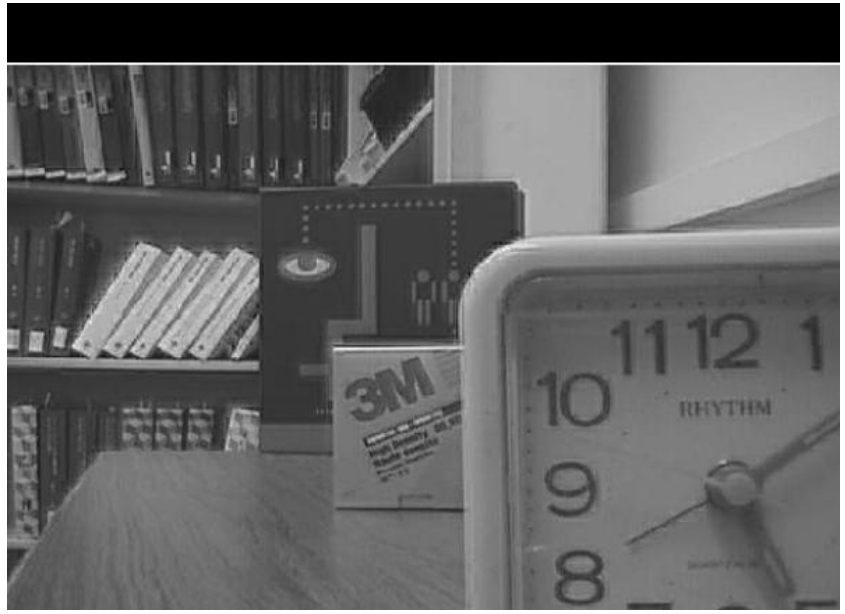

Figure-7: Fused Image

\section{CONCLUSION}

A technique used for fusing 2 dimensional multi-resolution 2-Dimensional pictures the use of wavelet transform below the accomplice slope with smoothens standard. The helpfulness of the approach have be illustrated the use of a variety of experimental picture couples such since the multifocus pictures, multi-sensor satellite picture and CT with MR picture of cross-section of person brain, qualitatively and fused image. The make use of of each slope and qualified smoothens situation ensure 2 double things. Whereas the slope criterion guarantee make sure that limits within the pictures be covered inside the fused algorithm, the absolute smoothens principle ensure so as to the area's of same depth also be integrated inside the fused picture accordingly the impact of noise is minimized. It have to survive distinctive with the intention of the deliberate algorithm is domain-independent. That useful resource makes use of statistics of neither the imaging system not the matters individual imaged. Hence, it is regularly utilized toward combination of a multiplicity of varieties of multimodal pictures. 2nd, because the real fusion is completed for the duration of the development of adapted coefficients, the method has been prolonged to combination of ' $\mathrm{N}$ ' pictures since by now planned within the algorithm.

PCNN neural network combining by multiresolution wavelet transform, a multi-resolution image fusion algorithm developed totally under adaptive connecting power PCNN be planned. In categorize in the direction of reproduce the cure sample of human eyes visible arrangement, PCNN connecting energy is modified through the exchange of nearby difference, this make's to the area of fusion picture be 386 constant, element attribute be within the excessive soft and visible result be fine. Every of these meant for the summarize picture cure with recognition provide a correct establishment.

\section{REFERENCES}

1. "Study of different image fusion algorithm," International Journal of Emerging Technology and Advanced Engineering, vol. 3, no. 5, pp. 288-291, 2013.

2. D. Yalamanda and V. P. Bhushan, "Medical image fusion based on improved wavelet transform."

3. K. Rani and R. Sharma, "Study of image fusion using discrete wavelet and multiwavelet transform."

4. V. Naidu and J. Raol, "Pixel-level image fusion using wavelets and principal component analysis,'Defence Science Journal, vol. 58, no. 3, pp. 338-352, 2008.

5. Wang, Wencheng and Chang, Faliang, "A multi-focus image fusion method based on Laplacian pyramid," Journal of Computers, 2011.

6. Li, Shutao and Kwok, JT-Y and Tsang, Ivor W and Wang, Yaonan, "Fusing images with different focuses using support vector machines," Neural Networks, IEEE Transactions on. IEEE, 2004, pp. 1555-1561

7. Liang, Junli and He, Yang and Liu, Ding and Zeng, Xianju, "Image fusion using higher order singular value decomposition," Image Processing, IEEE Transactions on, vol. 31, no. 12, pp. 2898-2909, 20122013.

8. Li, Shutao and Kang, Xudong and Hu, Jianwen, "Image fusion with guided filtering,',IEEE transactions on image processing: a publication of the IEEE Signal Processing Society, IEEE, vol. 27, no. 2, pp.2864-2875, 2013.

9. K. He, J. Sun, and X. Tang, "Guided image filtering," in Computer Vision-ECCV 2010. Springer, 2010, pp. 1-14.

10. Gu, W. Li, M. Zhu, and M. Wang, "Local edge-preserving multiscale decomposition for high dynamic range image tone mapping," Image Processing, IEEE Transactions on, vol. 22, no. 1, pp. 70-79, 2013.

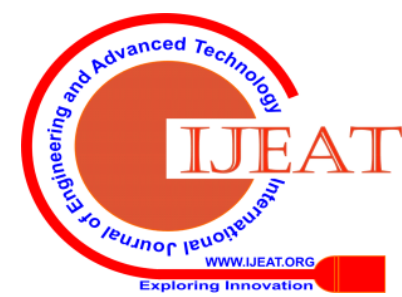

